\title{
A View from the Bridge
}

\author{
Natalie Paquette
}

$\mathrm{S}$

TRING THEORY is a quantum theory of gravity. ${ }^{1}$ Albert Einstein's theory of general relativity emerges naturally from its equations. ${ }^{2}$ The result is consistent in the sense that its calculations do not diverge to infinity. String theory may well be the only consistent quantum theory of gravity. If true, this would be a considerable virtue. Whether it is true or not, string theory is indisputably the source of profound ideas in mathematics. ${ }^{3}$ This is distinctly odd. A line of influence has always run from mathematics to physics. When Einstein struggled to express general relativity, he found the tools that he needed had been created sixty years before by Bernhard Riemann. The example is typical. Mathematicians discovered group theory long before physicists began using it. In the case of string theory, it is often the other way around. Physics has lent the dignity of its ideas to mathematics. The result is what Greg Moore has called physical mathematics. $^{4}$

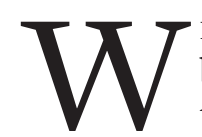

E OFTEN ENVISION physics on a flat spatial background. The billiard balls are round, but the table is flat. In performing experiments on the earth's surface, we treat the curvature of the planet as negligible, and take as our background three-dimensional Euclidean space. From the sphere to the torus and beyond, there are many more shapes on which to study physical systems. These offer different and fascinating ways of understanding physics. An electron confined to a sphere through which a magnetic flux passes can only occupy certain quantized energy levels. Similarly, a torus has two nontrivial cycles. The winding numbers of a string keep track of how many times it has wound itself around each cycle.

Quantum mechanics is one thing; special relativity, another. These theories are not naturally disposed to peaceful coexistence. Orthodox quantum mechanics makes no allowances for particle creation and annihilation. Special relativity encourages both. Fields are needed to handle the overflow. Quantum field theories are quantum-mechanical systems obeying special relativity. The Standard Model is a quantum field theory. Physicists often endow quantum field theories with extra symmetries. For example, supersymmetric theories require particles to come in pairs. For every bosonic particle there is a fermionic superpartner.

Supersymmetric field theory has a disheartening impediment. Suppose that a supersymmetric quantum field theory is defined on a generic curved manifold. The Euclidean metric of Newtonian physics and the Lorentz metric of special relativity are replaced by the manifold's own metric. Supercharges correspond to conserved Killing spinors. Solutions to the Killing spinor equations are plentiful in a flat space, but the equations become extremely restrictive on curved manifolds. They are so restrictive that they have, in general, no solutions. Promoting a flat supersymmetric field theory to a generic curved manifold breaks some or all of its supersymmetries. Calabi-Yau manifolds, as it happens, enjoy a certain flatness property-Ricci-flatness, a sort of flatness lite; and they admit conserved Killing spinors.

But the sphere admits no such solutions.

In the 1980s, Edward Witten introduced physicists to the topological twist. ${ }^{5}$ A twist works to couple a supersymmetric field to a curved manifold. With just the right twist, nontrivial solutions to the Killing spinor equations emerge. This is very much a salvage operation, one rescuing some fraction of the supersymmetries found in flat space. Physical observables of the twisted theory are just a subset of those that appear in the untwisted theory. While the observables in the untwisted theory depend, among other things, on the detailed geometry of the background manifold, the subset appearing in the twisted theory often depends only on its topological details.

This is important; it is mathematically important.

Topologically twisted field theories are sometimes called cohomological field theories. The twist endows such theories with a Grassmann, or anti-commuting, scalar symmetry Q. The physical observables lie in the cohomology of this symmetry. Deformations of the metric are Q-exact, and this immediately enforces the metric-independence of the theory's correlation functions. Correlation functions of Q-closed fields can sometimes be computed exactly by the powerful technique of supersymmetric localization. ${ }^{6}$ 
These computable correlation functions are topological or geometric invariants. Even if the physical universe somehow did not exist, these invariants would still be the cynosure of many mathematical eyes.

$\mathrm{F}$ OUR-DIMENSIONAL GEOMETRY encompasses a wealth of exotic structures. The mathematician's first imperative is to impose order on its abundance by classifying its four-dimensional manifolds. Not everything must be done at once. First things first. When are two manifolds topologically equivalent, or homeomorphic? In 1982, Michael Freedman showed that two manifolds are homeomorphic if and only if they have the same intersection form on their (co)homology lattices. ${ }^{7}$ Second things second. Homeomorphic manifolds may not be diffeomorphic; they are not equivalent as smooth manifolds. Smoothness imposes a new level of stratification among the manifolds, and with the new level, a new question. How to distinguish manifolds that are homeomorphic, but not diffeomorphic, from those that are both? In 1983, Simon Donaldson introduced a set of invariants of smooth 4-manifolds which distinguish homeomorphic-but-not-diffeomorphic manifolds. ${ }^{8}$ Donaldson invariants have a rigorous geometric definition, but they owed their inspiration to instanton configurations in Yang-Mills gauge theory. These configurations are solutions to the theory's equations of motion. Among mathematicians, they are known as anti-self-dual (ASD) connections.

A Lie group $\mathrm{G}$ is given, together with a principal G-bundle P over M. The connection is A, which is linked to the concept of parallel transport. Physicists refer to $\mathrm{A}$ as a gauge field, and, like all other fields, $\mathrm{A}$ is allowed to fluctuate in the path integral. There are other natural vector bundles $\mathrm{E}$ over $\mathrm{M}$. These are associated to representations of $\mathrm{G}$ using the principal G-bundle. Their connections are induced from A. Physicists regard them as matter fields. The curvature of A is a two-form called the gauge field strength, and it may be split into self-dual and anti-self-dual (ASD) components. If a field strength is purely ASD, then integration over $\mathrm{M}$ yields a positive integer, its instanton number. The ASD connections minimize the Yang-Mills action so that each instanton number labels a different topological sector, or component in the space of possible field configurations. For a fixed instanton number, there is an abstract geometric space of possible ASD connections-the instanton moduli space. In the simplest case, directions in the moduli space correspond to parameters such as the spatial position of an instanton.

Donaldson defined his topological invariants as integrals of differential forms. Integration over differential forms is no more demanding than advanced calculus, but what made Donaldson's application of such techniques so daring was his decision to compute these integrals in the moduli space of ASD connections. ${ }^{9}$ Donaldson also con- structed a map to obtain the appropriate differential forms from the homology of M.

In coming to understand the Donaldson invariants, physicists struck gold. They provided the practical computations, and several concepts required to complete the important proofs. Donaldson invariants over M may be folded into Donaldson-Witten generating functions. The Witten of Donaldson and Witten is Edward Witten, the only physicist to have won a Fields Medal.

In 1994, Witten introduced mathematicians to a twisted supersymmetric version of Yang-Mills, placing the theory on curved 4-manifolds. ${ }^{10}$ The result is Donaldson-Witten theory. Donaldson invariants appear as the correlation functions of the twisted Yang-Mills. Each correlation function serves to compute a coefficient in the Donaldson-Witten generating function. Witten showed explicitly how gauge-invariant polynomials of the topological field theories, as well as their Q-symmetries, generated all differential forms in the image of the Donaldson map.

Nathan Seiberg and Witten then undertook a beautiful physical study of supersymmetric gauge theories, and showed that their behavior is equivalent to a field theory describing weakly coupled monopoles. ${ }^{11}$ Equivalences between two different-looking physical systems are known as dualities. They are ubiquitous in field and string theory. One description of the system may be easy to study, but the other is usually not.

Seiberg and Witten's work led to a new set of computable geometric invariants called the Seiberg-Witten invariants, which count the solutions to monopole equations. ${ }^{12}$ These invariants, Witten demonstrated, convey all of the information provided by the Donaldson invariants, but their simple monopole description makes many properties of the Donaldson invariants as plain as day and eminently computable. In the space of a few weeks, Donaldson wrote, "long-standing problems were solved, new and unexpected results were found, along with simpler new proofs of existing ones, and new vistas for research opened up."13

This radical simplification of a deep and difficult set of mathematical ideas was an oblation from theoretical physics. The mathematicians never expected to get it, and the physicists never expected to give it.

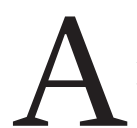
STRING IS extended in one spatial dimension and propagates itself in time. As it wriggles, the string sweeps out a two-dimensional surface in spacetime, its worldsheet. Field theories supported on the string worldsheet are both conformal and supersymmetric. Conformal symmetries are closely related to the symmetry of a system under scale invariance. Whether zoomed in or zoomed out, such systems remain the same.

Enumerative geometry concerns itself with counting solutions to natural geometric problems. In $200 \mathrm{BCE}$, Apollonius wondered how to find the number of circles 
tangent to three given circles in a plane. There are eight. Were Apollonius alive today he might well ask how many surfaces can be embedded inside a higher-dimensional manifold, such as a Calabi-Yau manifold.

A string propagating on a Calabi-Yau manifold may probe its geometry in a way that is sensitive to the number of its complex curves. The information is extraordinarily useful because it is precisely these numbers that enumerate the number of ways in which the string worldsheet can be embedded in the Calabi-Yau manifold. Consider the set of maps from a Riemann surface, or worldsheet, into a Calabi-Yau manifold X. The two-dimensional quantum field theory describing is called a supersymmetric non-linear sigma model. Two-dimensional bosonic fields have an interpretation as local coordinates on X. Fermionic or gauge fields map to sections of appropriate bundles, while the coupling constants in the action are geometric parameters associated to $\mathrm{X}$. The coupling constant for the bosonic kinetic term is just the metric on $\mathrm{X}$.

String theory might have much to say about enumerative geometry, the more so if it could isolate the data in the sigma model that encodes the number of curves on the manifold.

With this sort of extraction in mind, we might topologically twist the non-linear sigma model. Two topological twists are possible, the A-twist and B-twist, and with these twists, two theories, $\mathrm{A}(\mathrm{X})$ and $\mathrm{B}(\mathrm{X}){ }^{14}$ Both are topological field theories, comparable in their own way to Donaldson-Witten theory. Their correlation functions are independent of the metric on the two-dimensional world-sheet. On the other hand, these correlation functions have different space-time interpretations depending on the twist, each corresponding to a different subset of maps in the untwisted sigma model. The A-twist localizes the observables to the holomorphic maps on X; with the B-twist, localization selects the constant maps.

Although the two twists produce quite different-looking theories, it turns out that the difference between an A-twist and a B-twist is a matter of a minus sign. In the untwisted theories, there is an isomorphism between sigma models differing only in their sign. One sigma model has as its target a Calabi-Yau manifold, $\mathrm{X}$, the other, ${ }^{15} \mathrm{a}$ Calabi-Yau manifold $\mathrm{Y}^{16}$ The equivalence is called a mirror symmetry: $\mathrm{Y}$ is the mirror of $\mathrm{X}$. At the level of twisted theories, this equivalence becomes an identity $\mathrm{A}(\mathrm{X})=\mathrm{B}(\mathrm{Y})$. It turns out that since constant maps are simple to study and holomorphic maps less so, computing quantities in $\mathrm{B}(\mathrm{Y})$ is a powerful way to compute quantities in $\mathrm{A}(\mathrm{X})$.

These yield the Gromov-Witten invariants, which are directly related to counting curves. The power of mirror symmetry was first exhibited in the context of a simple Calabi-Yau manifold known as the quintic. ${ }^{17}$ Curve counts in manifolds can be stratified by the degree of the curve, and then assembled into a generating function that organizes the number of curves for each degree. The more complicated or intricate a curve, the higher its degree; and the number of curves proliferate as the degree grows. Curves of degree 1 are simply lines, and the number of (complex) lines in the quintic Calabi-Yau is easily computed. This was determined by Hermann Schubert in the late nineteenth century. There are 2,875 complex lines in the quintic. In 1986, Sheldon Katz determined that the quintic contains 609,250 curves of degree two.

Progress in enumerative geometry was slow. Computations quickly become laborious. If anyone was disposed to a brute-force count of curves of degree three, they kept their labors to themselves.

Philip Candelas et al. began studying string theory on the quintic in the early 1990s. They were guided by mirror symmetry. Denote the quintic by $\mathrm{X}$ and its mirror by $\mathrm{Y}$. Consider $\mathrm{A}(\mathrm{X})$. The Lagrangian, save one term, is Q-exact and therefore trivial in the cohomology of observables. The remaining term is an integral of the Kähler form, and the Kähler form is, as one might expect, a differential form. It is this form that allows one to measure volumes of cycles in, say, a Calabi-Yau manifold of X. A(X) depends only on the Kähler form. Correlation functions in $\mathrm{A}(\mathrm{X})$ reduce to integrals over the space of holomorphic maps, and these coincide with Gromov-Witten invariants. The sigma model requires an infinite series of difficult non-perturbative corrections. By the magic of mirror symmetry, they must be equivalent to quantities in $\mathrm{B}(\mathrm{Y})$, and these reduce to integrals over the space of constant maps. These integrals turn out to be classically exact quantities called periods, which depend on the complex structure of $\mathrm{Y}^{18}$ The Kähler structure controls the size of the manifold or submanifolds, the complex structure, its shape. Candelas et al. were able to compute the period integrals of $\mathrm{Y}$, and, using a judicious change of variables called the mirror map, expand the answer to extract Gromov-Witten invariants, order by order, in the degree of the holomorphic map.

There now followed a dazzling display of physical mathematics.

At the same time Candelas et al. were wandering down a hall of mirrors, mathematicians were working hard to count curves of degree three using their own sophisticated machinery and a variety of ingenious computer algorithms. Geir Ellingsrud and Stein Strømme conjectured that there were 2,682,549,425 such curves. Analytical methods of computation and proof had, at this stage, lapsed. Brute force methods prevailed. They presented this result at the Mathematical Sciences Research Institute in Berkeley. It was 1991. Candelas and his collaborators demurred. The number was $317,206,375$. The mathematicians were skeptical. In mirror symmetry, the physicists were using a mathematically unheard-of technique. Their computations came hand in hand with the remarkable claim that classical period integrals in one Calabi-Yau were equivalent to curve counts in an entirely different Calabi-Yau. These claims were, if true, revolutionary. Ellingsrud and 
Strømme meticulously checked their work and found a bug in their computer code.

They announced their correction in a lapidary telegram: "Physics Wins!" 19

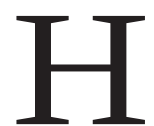

IGH ENERGY PHYSICISTS organize theories by their symmetries. Supersymmetry and conformal symmetry are examples. Many aspects of a physical theory, like the spectrum of its particle-like excitations, are constrained by requiring them to be compatible with the system's symmetries. Group theory is everywhere.

The finite groups contain a finite number of elements. In general, the finite groups may be decomposed into subnormal series, where each group in the sequence is a normal subgroup of the next. Finite simple groups are finite groups with no nontrivial normal subgroups. They are the building blocks of the finite groups. The finite simple groups are analogous to the prime numbers. As soon as the finite groups were understood, mathematicians conceived the desire to classify them, and after decades spent in painstaking, and tedious, collaboration, in 2004, they did. The finite simple groups fall into eighteen infinite families of well-understood groups such as the cyclic groups of prime order, and twenty-six exotic or sporadic groups. Of the sporadic groups, the largest is the Monster, which contains roughly $10^{54}$ elements. Many of the other sporadic groups can be realized as subquotients of this behemoth. The sporadic groups are exceptional structures; whether they would play any deeper role in mathematics remained unclear.

The answer to this question turned out to be contingent on the way in which the Monster was represented. A representation expresses an abstract group in terms of linear actions on a vector space, and thereby associates each element of an abstract group to a matrix. The size of the matrix is the dimension of the representation. The irreducible representations form a complete set of indecomposable representations from which all other representations may be built by simple operations like direct summation. The Monster has 194 irreducible representations. Every group has a one-dimensional representation corresponding to the trivial group action. After the trivial representation, the second smallest irreducible representation of the Monster is 196,883 dimensional, the next smallest, 21,296,876 dimensional, and so on. These are not numbers that encourage the mathematician to think in terms of explicit constructions. The Monster, and the natural objects on which it acted, remained mysterious until the advent of monstrous moonshine.

Modular forms arise very naturally in number theory. They are functions $f(\tau)$ defined on the complex upper half-plane. They transform covariantly when $\tau$ is acted upon by an element $\gamma$ of the modular group $\mathrm{SL}_{2}(\mathrm{Z}): f(\gamma \cdot \tau)=(c \tau+d)^{k} f(\tau)$. This is the group of $2 \times 2$ matrices with integral entries and unit determinant. The half-integer $k$ is called the weight of the modular form, and $c$ and $d$ represent the two integer entries on the lower row of the matrix $\gamma$. Modular forms are important mathematical objects. The coefficients in the expansions of such forms are often integers of interest to number theorists. Proof of these integer-identities is sometimes derived from functional identities antecedently satisfied by modular form..$^{20}$ The $\mathrm{j}$-function is a special function invariant under modular transformations: it transforms as a modular form of weight 0 . The j-function is, in fact, the generator of all such modular-invariant functions since they are all expressible as ratios of polynomials of the $\mathrm{j}$-function.

There now follows one of those enlightening surprises that are characteristic of physical mathematics, one first noted by the group theorist John McKay in 1978. In idly reading a number theory book, he came across the j-function and observed that its Fourier expansion starts with some interesting coefficients-first 1, and then 196,884. But 196,884 is $1+196,883$, and this is the dimensional sum of the first two irreducible representations of the Monster. He wrote to John Thompson, who observed that the next coefficient of the $\mathrm{j}$-function is $21,493,760=21,296,876+$ $196,883+1$.

Could this elegant number theoretic object know something about the largest sporadic simple group? It seemed shocking and bizarre. Whence the phrase, monstrous moonshine.

The mathematicians John Conway and Simon Norton first codified monstrous moonshine by asking how a distinguished class of modular objects could encode data about the Monster. ${ }^{21}$ They conjectured that one could associate a modular function to each conjugacy class of the Monster that was invariant with respect to transformations under special, genus zero subgroups $\mathrm{G}$ of $\mathrm{SL}_{2}(\mathrm{R}){ }^{22}$ If so, their Fourier expansions might contain representation-theoretic data about the Monster. Their coefficients would then be the characters of the group element, and the modular function associated with the identity class, the j-function. ${ }^{23}$

Collective conjectures became known as monstrous moonshine.

And they were proved by Richard Borcherds in 1992. ${ }^{24}$ Some elements of his proof were directly inspired by string theory. He also introduced many new mathematical structures, generalized Kac-Moody algebras among them, and these in turn have led back to interesting physics. ${ }^{25}$ Much of the physical content of the monstrous moonshine conjectures, and the central ingredient in Borcherds's proof, comes from the mathematician's refinement of conformal field theory. In mathematics, conformal field theories are known as vertex operator algebras. ${ }^{26}$ The vertex operator algebra that clarifies monstrous moonshine was constructed by Igor Frenkel, James Lepowsky, and Arne Meurman..$^{27}$ The back translation into string theory was 
undertaken by Lance Dixon, Paul Ginsparg, and Jeffrey Harvey. ${ }^{28}$ For string theorists, the $\mathrm{j}$-function is a bookkeeping device, a partition function that counts particle states at a given energy level. The Monster acts on this vertex operator algebra as a symmetry. It commutes with the Hamiltonian and preserves the ground state, while excitations above the vacuum are organized into representations of the symmetry.

The modularity of the partition function is physically natural. Consider a conformal field theory on the worldsheet of a closed string loop. The worldsheet has the topology of a cylinder. To compute the partition function, the two ends of the cylinder are fused to form a torus. The Euclidean time coordinate plays the role of a finite temperature-a standard identification in quantum mechanics and quantum field theory. The modular group $\mathrm{SL}_{2}(\mathrm{Z})$ is the group of symmetries of the torus viewed as a topological space, and so the group assigned to classes of homeomorphisms mapping the torus to itself. ${ }^{29}$ These symmetries do not affect the underlying physics. This is the string theoretic promotion of the familiar fact that nothing we compute about a point particle in quantum mechanics depends on the parameterization of its worldline. A respect for physical consistency demands that an arbitrary parameterization of the torus cannot affect an observable quantity like the partition function. The partition function must be a modular invariant under $\mathrm{SL}_{2}(Z)$.

The other modular functions of monstrous moonshine, associated with genus zero subgroups of $\mathrm{SL}_{2}(\mathrm{R})$, also have conformal field theoretic interpretations when the genus zero group is a subgroup of $\mathrm{SL}_{2}(Z)$. Their modularity falls under the purview of the physical argument just given. For genus zero subgroups of $\mathrm{SL}_{2}(\mathrm{R})$ not contained in $\mathrm{SL}_{2}(\mathrm{Z})$, the modularity of the relevant functions had no obvious explanation, physical or mathematical. Borcherds, of course, proved the conjectures, but this aspect of his proof involved a brute-force verification, as opposed to a conceptual explanation, of the genus zero property. This has long been an outstanding mystery in the already-mysterious moonshine. Just recently, Daniel Persson, Roberto Volpato and I proposed a conceptual explanation for the genus zero property of monstrous moonshine using the space-time properties of a heterotic string theory. ${ }^{30}$ This construction also placed the algebraic ingredients of Borcherds's proof firmly in a physical context.

The monstrous moonshine observations are ultimately natural consequences of string theoretic spacetime and worldsheet symmetries and have generated striking and far-flung algebraic structures. Many years ago, Eugene Wigner asked for an explanation of the unreasonable effectiveness of mathematics within physics. His essay was influential more for asking the question than for answering it. It would be possible today to write a comparable essay asking for the explanation of the unreasonable effectiveness of physics within mathematics. If mathemat- ics and physics are in so many respects in equipoise, then the differences between them may be less a matter of their content than their technique; and that, in the end, they serve to show that there is only one reality to which they both appeal.

Wouldn't it be lovely to think so?

Natalie Paquette is a Sherman Fairchild Postdoctoral Scholar in Theoretical Physics at the California Institute of Technology.

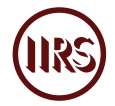

1. For a discussion of string theory aimed at a general audience, see: Brian Greene, The Elegant Universe: Superstrings, Hidden Dimensions, and the Quest for the Ultimate Theory (New York: Vintage, 1999).

2. Joseph Polchinski, String Theory Volume 1: An Introduction to the Bosonic String (Cambridge: Cambridge University Press, 1998); Joseph Polchinski, String Theory Volume 2: Superstring Theory and Beyond (Cambridge: Cambridge University Press, 1998).

3. While I believe theoretical physics is uniquely empowered to raise deep mathematical questions, this does not suggest a dearth of progress in the opposite direction, though that is outside of my topic here. Increased mathematical sophistication has taught us beautiful truths about theoretical physics, and I am always personally heartened when my own research points towards mathematical concepts of especial depth and elegance. This cross-cultural knowledge is often a crude but useful signpost when studying theoretical structures. The appearance of Riemannian geometry in Einstein's general relativity is one prominent example of mathematical beauty far preceding experimental confirmation, but, nonetheless, helping guide the way.

4. For a definition and survey of physical mathematics and an inspiring collection of references and examples, see Gregory Moore, "Physical Mathematics and the Future," Vision Talk, Strings2014, Princeton, June 27, 2014.

5. Edward Witten, "Constraints on Supersymmetry Breaking," Nuclear Physics B 202, no. 2 (1982): 253-316; Edward Witten, "Supersymmetry and Morse Theory," Journal of Differential Geometry 17, no. 4 (1982): 661-92; Edward Witten, "Topological Quantum Field Theory," Communications in Mathematical Physics 117, no. 3 (1988): 353-86; Edward Witten, "Introduction to Cohomological Field Theories," International Journal of Modern Physics A 6, no. 16 (1991): 2,775-92.

6. For a recent review, see Vasily Pestun et al., "Localization Techniques in Quantum Field Theories," Journal of Physics A: Mathematical and Theoretical 50, no. 440,301 (2017).

7. This holds for simply connected, smooth four-manifolds. See Michael Freedman, "The Topology of Four-Dimen- 
sional Manifolds," Journal of Differential Geometry 17, no. 3 (1982): 357-453.

8. Simon Donaldson, "An Application of Gauge Theory to Four-Dimensional Topology," Journal of Differential Geometry 18, no. 2 (1983): 279-315; Simon Donaldson, "Self-Dual Connections and the Topology of Smooth 4-Manifolds," Bulletin of the American Mathematical Society 8, no. 1 (1983): 81-83. For an overview and more complete references, see Simon Donaldson and Peter Kronheimer, The Geometry of Four-Manifolds (Oxford: Oxford University Press, 1990).

9. I have, of course, omitted many technical details. More precisely, one studies the moduli space of irreducible ASD connections, so that the isotropy group of a connection is the center of $\mathrm{G}$.

10. Edward Witten, "Supersymmetric Yang-Mills Theory on a Four-Manifold," Journal of Mathematical Physics 35 (1994): 5,101-35.

11. Nathan Seiberg and Edward Witten, "Electric-Magnetic Duality, Monopole Condensation, and Confinement in $N=2$ Supersymmetric Yang-Mills Theory," Nuclear Physics B 426, no. 1 (1994): 19-52.

12. Edward Witten, "Monopoles and Four-Manifolds," (1994), arXiv:hep-th/9411102.

13. For both the quote and a survey of these developments, see Simon Donaldson, "The Seiberg-Witten Equations and 4-Manifold Topology," Bulletin of the American Mathematical Society 33 (1996): 45-70.

14. Edward Witten, "Topological Sigma Models," Communications in Mathematical Physics 118, no. 3 (1988): 411-49. For a review see Edward Witten, "Mirror Manifolds and Topological Field Theory,” (1991), arXiv:hep-th/9112056.

15. More precisely, the sign flip is a choice of convention for charges under a conserved U(1) current, which is one generator in the worldsheet superconformal algebra.

16. One among many topological identities between $X$ and $Y$ : the Hodge numbers of $\mathrm{X}$ can be obtained from those of $\mathrm{Y}$ by a reflection along the diagonal of the Hodge diamond.

17. Philip Candelas et al., "A Pair of Calabi-Yau Manifolds as an Exactly Soluble Superconformal Theory," Nuclear Physics B 359, no. 1 (1991): 21-74.

18. Again, one must study which quantities in the Lagrangian or correlation functions are Q-exact with respect to the Q obtained from B-twist.

19. For a more detailed account of these events for a general audience, see Shing-Tung Yau and Steve Nadis, The Shape of
Inner Space: String Theory and the Geometry of the Universe's Hidden Dimensions (New York: Basic Books, 2010).

20. Modular forms were central to Andrew Wiles's proof of Fermat's last theorem: Andrew Wiles, "Modular Elliptic Curves and Fermat's Last Theorem," Annals of Mathematics 141, no. 3 (1995): 443-551. A more recent proof using modular forms to great effect can be found in Maryna Viazovska, "The Sphere Packing Problem in Dimension 8,” (2016), arXiv:1603. 04246.

21. John Conway and Simon Norton, "Monstrous Moonshine," Bulletin of the London Mathematical Society 11, no. 3 (1979): 308-39.

22. This name comes from the fact that the quotient of the upper half-plane by the modular group $\mathrm{G}$ has the topology of a sphere. A special property of genus zero groups, like $\mathrm{SL}_{2}(Z)$, is that there is a modular function that serves as a canonical generator for the other modular functions with respect to that group, like the $\mathrm{j}$-function. The modular functions appearing in monstrous moonshine are all of this type.

23. Characters are simply ordinary traces of the given group element in the prescribed representation. The trace operation, being cyclic, depends only on the conjugacy class of the element.

24. Richard Borcherds, "Monstrous Moonshine and Monstrous Lie Superalgebras," Inventiones Mathematicae 109, no. 1 (1992): 405-44.

25. Jeffrey Harvey and Gregory Moore, "Algebras, BPS States, and Strings," Nuclear Physics B 463, no. 2-3 (1996): 315-68.

26. More precisely, a vertex operator algebra is the formalization of the notion of a holomorphic, or chiral, conformal field theory. Conformal field theories in physics are typically combined with an anti-holomorphic sector as well.

27. Igor Frenkel, James Lepowsky, and Arne Meurman, Vertex Operator Algebras and the Monster (Cambridge, MA: Academic Press, 1989).

28. Lance Dixon, Paul Ginsparg, and Jeffrey Harvey, "Beauty and the Beast: Superconformal Symmetry in a Monster Module," Communications in Mathematical Physics 119, no. 2 (1988): 221-41.

29. More precisely, it is the mapping class group.

30. Natalie Paquette, Daniel Persson, and Roberto Volpato, "BPS Algebras, Genus Zero, and the Heterotic Monster," (2017), arXiv:1701.05169; Natalie Paquette, Daniel Persson, and Roberto Volpato, "Monstrous BPS-Algebras and the Superstring Origin of Moonshine,” (2016), arXiv:1601.05412. 